\title{
Necessity of Focus on the Disaster Risk Perception: Lessons From the 2019 Floods in Iran
}

\author{
Hamid Safarpour, PhD (10; Moslem Sarani, PhD; Meysam Safi-Keykaleh, PhD
}

To the Editor,

The first priority for action in Sendai Framework for disaster risk reduction is understanding disaster risk. ${ }^{1}$ Risk perception affects risk management and, therefore, determines whether risk management is successful in reducing vulnerability. ${ }^{2}$ The degree of preparedness and response to risks is substantially determined by the quality and quantity of the information and the difference between the individuals' risk perceptions. Risk perception is a determining factor in the behaviors displayed in response to risks. ${ }^{3}$

Awareness, worry, and preparedness are the core elements of risk perception. ${ }^{4}$ As an important component of adaptation to risks, awareness is vital for society. Worry is also an essential trait and a normative value for awareness. Higher levels of worry increase the probability of preparedness. Furthermore, worry plays a major role in the relationship between awareness and preparedness. ${ }^{4}$ Risk perception generally consists of 2 chief dimensions: the cognitive dimension, which is linked to people's awareness and understanding of risk; and the emotional dimension, which is associated with people's perception of the risk. ${ }^{5}$

Wachinger et al. (2013) classified the disaster risk perception factors into 4 categories: the risk factors, informational factors, personal factors, and contextual. ${ }^{6}$ Hence, disaster risk perception is determined by various factors, and the knowledge of these factors contributes to risk management in society. Moreover, despite the advances in disaster risk management, disaster risk perception is still one of the important determinants of preparedness and response to disasters.

The recent floods in Iran are important examples of these disasters. Approximately 25 provinces of Iran were inundated by floods in March 2019. Despite the warnings given by the Meteorological Organization of Iran about the inclement weather conditions and the high probability of flood in most parts of Iran at least 2 days before the arrival of the precipitation event and despite the warnings issued in mass media and the social networks, people neglected these warnings and kept traveling to and staying in the high-risk areas.
The flood in Shiraz killed 21, Lorestan 15, Golestan 8, Hamedan 8, Mazandaran 6, Khozestan 6, North Khorasan 3, Ghazvin 2, Kohkiloieh and Boyerahmad 2, Razavi Khorasan 1, Ghom 1, Semnan 1, Kermanshah 1, and in Ilam 1 people, respectively. As a result of these floods, a total of 76 people died by April 14, 2019.7,8

There were numerous examples of the people's low-risk perception. In Shiraz, the streets were flooded in the morning while the police had severely warned the residents and the travelers in advance. However, people ignored the police warnings and entered the risk zone. Although the rescue teams had closed the road to Darvazeh Quran and had asked all drivers to leave the area, some of them refused to cooperate and disrupted the traffic flow. Some drivers were also unwilling to listen to the relief and rescue teams and insisted on going on. After a few hours, the area was in full flood stage, which dislocated all the automobiles and killed or injured several people. This flash flood occurred within $15 \mathrm{~min}$, but it led to irreversible consequences, which were partly caused by the lack of public risk perception.

Furthermore, many people took unsafe or neglectful actions after perceiving the flood risk. For instance, some people were taking pictures and movies of severe floods or taking selfies in high-risk areas. Some others also entered the flooded areas out of curiosity instead of running for shelter. With regard to the flood in Khuzestan, when the Dez Dam burst due to the severe precipitation, the dam gate was opened to control the flood. However, people invaded the outlet of the gate regardless of the flood risk to catch fish. After Lorestan Province was struck by the flood, people were waiting for a bridge to collapse in a highly risky zone and started filming and watching the bridge. In Darreh Shahr city of Ilam Province, when the city bridge collapsed, people entered the highly hazardous river banks to watch the scene and take pictures. These behaviors are due to a lack of disaster risk perception.

The following suggestions are provided for improving the disaster risk perception based on information gained from recent incidents: 
- Improving the disaster risk perception by planning in different areas, including education, research, health, and culture, with an emphasis on the social groups, especially families.

- Launching national and international campaigns to raise public awareness about the risk of disasters and providing training to promote the disaster prevention culture, increase national resilience and citizens' accountability, increase the public's understanding and perception of risks, and mutually support the spread of the disaster risk knowledge and experience.

- Encouraging the public and private sectors to actively participate in these campaigns on the local, national, and international levels.

- Valuing the role of risk communications in the management of emergencies and disasters and modifying the effective risk communication methods by establishing trust, giving early warnings, ensuring transparency, respecting public concerns and worries, and planning for the existing or expected problems.

- Increasing the availability of knowledge and technologies and developing them by carrying out fundamental and applied research to improve the risk perception and manage the risk of emergencies and disasters more effectively.

\section{About the Authors}

Department of Nursing, Faculty of Nursing and Midwifery, Ilam University of Medical Sciences, Ilam, Iran (Dr Safarpour) and Department of Health in Disasters and Emergencies, School of Public Health and Safety, Shahid Beheshti University of Medical Sciences, Tehran, Iran (Dr Safarpour; Dr Sarani; Dr Safi-Keykaleh).
Correspondence and reprint requests to Meysam Safi-Keykaleh, Department of Health in Disasters and Emergencies, School of Public Health and Safety, Shahid Beheshti University of Medical Sciences, Tehran, Iran (e-mail: meysam.safi@ yahoo.com).

\section{REFERENCES}

1. UNISDR (United Nations International Strategy for Disaster Reduction). Sendai framework for disaster risk reduction 2015-2030. 2015. http://www. wcdrr.org/uploads/Sendai_Framework_for_Disaster_Risk_Reduction_20152030.pdf. Accessed January 28, 2020.

2. Bubeck P, Botzen WJ, Aerts JC. A review of risk perceptions and other factors that influence flood mitigation behavior. Risk Anal. 2012;32(9): 1481-1495.

3. Safi Keykaleh M, Khorasani Zavareh D, Safarpour H, et al. Different perception, different behavior, necessity of consensus over "risk" term. Health Emerg Disasters Q. 2018;3(3):121-122.

4. Bradford R, O'Sullivan J, Van der Craats I, et al. Risk perception-issues for flood management in Europe. Nat Hazards Earth Syst Sci. 2012;12(7): 2299-2309.

5. Paek H-J, Hove T. Risk perceptions and risk characteristics. Oxford Research Encyclopedia of Communication. 2017. https://oxfordindex.oup. com/view/10.1093/acrefore/9780190228613.013.283. Accessed January 28, 2020.

6. Wachinger $\mathrm{G}$, Renn $\mathrm{O}$, Begg $\mathrm{C}$, et al. The risk perception paradoximplications for governance and communication of natural hazards. Risk Anal. 2013;33(6):1049-1065

7. Iranian Legal Medicine Organization (ILMO). 2019. http://www.lmo.ir. Accessed April 14, 2019.

8. Peyravi M, Marzaleh MA. Celebrities' effective presence during the large flood in Iran in 2019. Prehosp Disaster Med. 2019;34:681-682. 